\title{
Signless Laplacian spectral radius and Hamiltonicity of graphs with large minimum degree
}

\author{
Yawen $\mathrm{Li}^{*} \quad$ Yao Liu ${ }^{\dagger} \quad$ Xing Peng ${ }^{\ddagger}$
}

\begin{abstract}
In this paper, we establish a tight sufficient condition for the Hamiltonicity of graphs with large minimum degree in terms of the signless Laplacian spectral radius and characterize all extremal graphs. Moreover, we prove a similar result for balanced bipartite graphs. Additionally, we construct infinitely many graphs to show that results proved in this paper give new strength for one to determine the Hamiltonicity of graphs.
\end{abstract}

\section{Introduction}

A Hamiltonian cycle in a graph is a cycle that visits all vertices. It is well known that the problem of determining the Hamiltonicity of graphs is remarkably difficult [7]. Thus it is meaningful to find sufficient conditions for graphs to be Hamiltonian. A seminal result due to Dirac [3] states that if $\delta(G)>\frac{n}{2}$, then $G$ is Hamiltonian, here $\delta(G)$ is the minimum degree of $G$ and $n$ is the number of vertices of $G$.

While many known results are in terms of vertex degrees and the number of edges, Krivelevich and Sudakov showed the following breakthrough result using eigenvalues of the adjacency matrix. Namely, they proved that a $d$-regular graph is Hamiltonian if its second largest eigenvalue (in the absolute value) of the adjacency matrix is sufficiently less than d. Butler and Chung [2] extended their result to general graphs by using eigenvalues of the combinatorial Laplacian matrix. Both of two results mentioned above used the Pośa's rotation method together with some discrepancy and discrete isoperimetric inequalities of graphs.

In 2009, Fiedler and Nikiforov [5] gave lower bounds on $\lambda(G)$ that imply the Hamiltonicity of $G$, where $\lambda(G)$ is the largest eigenvalue of the adjacency matrix of $G$. After that, many other spectral conditions in the same spirit for the Hamiltonicity of graphs have been discovered, see e.g., 11, 8, 9, 10, 11, 13. In this paper, we will first establish a signless Laplacian analogue of a result due to Nikiforov [10] for graphs with large minimum degree. Then we strengthen a result by Li and Ning [8] for balanced bipartite graphs. Before we state our theorems, we briefly recall results from [10. We need to introduce two families of graphs.

We use $K_{n}$ and $\bar{K}_{n}$ to denote the complete graph with $n$ vertices and the edgeless graph with $n$ vertices respectively. For two vertex disjoint graphs $G$ and $H$, we write $G+H$ for their disjoint union which satisfies $V(G+H)=V(G) \cup V(H)$ and $E(G+H)=E(G) \cup E(H)$. We write $G \vee H$ for their join which satisfies $V(G \vee H)=V(G) \cup V(H)$ and $E(G \vee H)=$ $E(G) \cup E(H) \cup\{x y: x \in V(G), y \in V(H)\}$.

\footnotetext{
${ }^{*}$ Center for Applied Mathematics, Tianjin University, Tianjin, 300072 China, (yawenli@tju.edu.cn),

${ }^{\dagger}$ Center for Applied Mathematics, Tianjin University, Tianjin, 300072 China, (liuyao@tju.edu.cn),

$\ddagger$ Center for Applied Mathematics, Tianjin University, Tianjin, 300072 China, (x2peng@tju.edu.cn), Research is supported in part by National Natural Science Foundation of China (No. 11601380).
} 
First, for any $k>1$ and $n \geq 2 k+1$, let

$$
M_{k}(n)=K_{k} \vee\left(K_{n-2 k}+\bar{K}_{k}\right) .
$$

The graph $M_{k}(n)$ is obtained from $K_{n-k}$ and $\bar{K}_{k}$ by connecting all vertices of $\bar{K}_{k}$ to all vertices of a $k$-subset of $K_{n-k}$.

Second, for any $k \geq 1$ and $n \geq k+2$, let

$$
L_{k}(n)=K_{1} \vee\left(K_{n-k-1}+K_{k}\right) .
$$

The graph $L_{k}(n)$ is obtained from $K_{n-k}$ and $K_{k+1}$ by identifying a vertex. We note for any admissible $k$ and $n$, graphs $L_{k}(n)$ and $M_{k}(n)$ are not Hamiltonian. Strengthening a result by Li and Ning [8, Nikiforov [10] proved the following theorem.

Theorem 1 Let $k>1, n \geq k^{3}+k+4$, and let $G$ be a graph of order $n$, with minimum degree $\delta(G) \geq k$. If

$$
\lambda(G) \geq n-k-1,
$$

then $G$ has a Hamiltonian cycle unless $G=M_{k}(n)$ or $G=L_{k}(n)$.

For a graph $G$, let $Q(G)=D+A$ be the signless Laplacian matrix, here $D$ is the diagonal matrix of degrees. We use $q(G)$ to denote the largest eigenvalue of $Q(G)$. If $E^{\prime} \subseteq E(G)$, then we will use $G-E^{\prime}$ to denote the subgraph of $G$ by deleting edges from $E^{\prime}$. We shall prove a signless Laplacian analogue of Theorem 1 To state our theorem, we need to define a collection of subgraphs of $M_{k}(n)$ and $L_{k}(n)$.

For the graph $M_{k}(n)$, let $X=\left\{v \in V\left(M_{k}(n)\right): d(v)=k\right\}, Y=\left\{v \in V\left(M_{k}(n)\right): d(v)=\right.$ $n-1\}$, and $Z=\left\{v \in V\left(M_{k}(n)\right): d(v)=n-k-1\right\}$. Let $E_{1}\left(M_{k}(n)\right)$ be the set of those edges of $M_{k}(n)$ whose both endpoints are from $Y \cup Z$. We define

$$
\mathcal{M}_{1}(n, k)=\left\{G \subseteq M_{k}(n): G=M_{k}(n)-E^{\prime}, \text { where } E^{\prime} \subset E_{1}\left(M_{k}(n)\right) \text { with }\left|E^{\prime}\right| \leq\left\lfloor\frac{k^{2}}{4}\right\rfloor\right\}
$$

Similarly, for the graph $L_{k}(n)$, we let $X=\left\{v \in V\left(L_{k}(n)\right): d(v)=k\right\}, Y=\{v \in$ $\left.V\left(L_{k}(n)\right): d(v)=n-1\right\}$, and $Z=\left\{v \in V\left(L_{k}(n)\right): d(v)=n-k-1\right\}$. It is clear that the set $Y$ contains only one point. We use $E_{1}\left(L_{k}(n)\right)$ to denote edges of $L_{k}(n)$ whose both endpoints are from $Y \cup Z$. We define

$$
\mathcal{L}_{1}(n, k)=\left\{G \subseteq L_{k}(n): G=L_{k}(n)-E^{\prime}, \text { where } E^{\prime} \subset E_{1}\left(L_{k}(n)\right) \text { with }\left|E^{\prime}\right| \leq\left\lfloor\frac{k}{4}\right\rfloor\right\} .
$$

Our first result is the following theorem.

Theorem 2 Assume $k>1$ and $n \geq k^{4}+k^{3}+4 k^{2}+k+6$. Let $G$ be a connected graph with $n$ vertices and minimum degree $\delta(G) \geq k$. If

$$
q(G) \geq 2(n-k-1)
$$

then $G$ has a Hamilton cycle unless $G \in \mathcal{M}_{1}(n, k)$ or $G \in \mathcal{L}_{1}(n, k)$.

To show Theorem 2 does not covered by Theorem 1, in the last section, we will construct infinitely many Hamiltonian graphs that satisfy the condition in Theorem 2 but do not satisfy the condition in Theorem [1. The proof of Theorem 2 relies on techniques in 8 and the following two lemmas.

Lemma 1 Assume $k>1$ and $n \geq k^{4}+k^{3}+4 k^{2}+k+6$. For each $G \in \mathcal{M}_{1}(n, k) \cup \mathcal{L}_{1}(n, k)$, we have $q(G) \geq 2(n-k-1)$. 
We will need another family of subgraphs of $M_{k}(n)$ and $L_{k}(n)$ defined as follows:

$$
\begin{aligned}
& \mathcal{M}_{2}(n, k)=\left\{G \subset M_{k}(n): G=M_{k}(n)-E^{\prime}, \text { where } E^{\prime} \subset E_{1}\left(M_{k}(n)\right) \text { with }\left|E^{\prime}\right|=\left\lfloor\frac{k^{2}}{4}\right\rfloor+1\right\} . \\
& \mathcal{L}_{2}(n, k)=\left\{G \subset L_{k}(n): G=L_{k}(n)-E^{\prime}, \text { where } E^{\prime} \subset E_{1}\left(L_{k}(n)\right) \text { with }\left|E^{\prime}\right|=\left\lfloor\frac{k}{4}\right\rfloor+1\right\} .
\end{aligned}
$$

We have the following lemma.

Lemma 2 Assume $k>1$ and $n \geq k^{4}+k^{3}+4 k^{2}+k+6$. For each $G \in \mathcal{M}_{2}(n, k) \cup \mathcal{L}_{2}(n, k)$, we have $q(G)<2(n-k-1)$.

In order to state our second theorem on balanced bipartite graphs, we need to introduce one more family of graphs. A bipartite graph is called balanced if its vertex parts have the same size. For $k>1$ and $n \geq 2 k$, we write $B_{k}(n)$ for the graph obtained from $K_{n, n}$ by deleting all edges in a subgraph $K_{k, n-k}$. We note $B_{k}(n)$ is not Hamiltonian. Li and Ning [8] proved the following theorem.

Theorem 3 Let $G$ be a balanced bipartite graph of order $2 n$ and of minimum degree $\delta(G) \geq$ $k>1$.

1. If $n \geq(k+1)^{2}$ and $\lambda(G) \geq \lambda\left(B_{k}(n)\right)$, then $G$ is Hamiltonian unless $G=B_{k}(n)$.

2. If $n \geq(k+1)^{2}$ and $q(G) \geq q\left(B_{k}(n)\right)$, then $G$ is Hamiltonian unless $G=B_{k}(n)$.

For the graph $B_{k}(n)$, let $S$ and $T$ be the vertex parts such that the degree of vertices from $T$ is either $n$ or $n-k$. Let $X=\{v \in S: d(v)=k\}, Y=\{v \in T: d(v)=n\}$, $W=\{v \in T: d(v)=n-k\}$, and $Z=\{v \in S: d(v)=n\}$. We note $S=X \cup Z$ and $T=Y \cup W$. We define $E_{1}\left(B_{k}(n)\right)$ as those edges of $B_{k}(n)$ whose both endpoints are from $Y \cup W \cup Z$. Let

$$
\mathcal{B}_{1}(n, k)=\left\{G \subseteq B_{k}(n): G=B_{k}(n)-E^{\prime}, \text { where } E^{\prime} \subseteq E_{1}\left(B_{k}(n)\right) \text { with }\left|E^{\prime}\right| \leq\left\lfloor\frac{k^{2}}{4}\right\rfloor\right\} .
$$

We strengthen Part 2 of Theorem 3 as follows.

Theorem 4 Assume $k>1$ and $n \geq k^{4}+3 k^{3}+5 k^{2}+5 k+4$. Let $G$ be a balanced bipartite graph with $2 n$ vertices and minimum degree $\delta(G) \geq k$. If

$$
q(G) \geq 2 n-k,
$$

then $G$ has a Hamilton cycle unless $G \in \mathcal{B}_{1}(n, k)$.

Here we notice $q\left(K_{n, n-k}\right)=2 n-k$ and $q\left(B_{k}(n)\right)>2 n-k$ since it contains $K_{n, n-k}$ as a proper subgraph. Thus the condition in Theorem 4 is weaker than the one in Part 2 of Theorem 3. This is the reason why Theorem 4 involves a larger family of exception graphs than Part 2 of Theorem 3 does. To show Theorem 4 is not covered by Part 1 of Theorem 3. in the last section, we will construct infinitely many Hamiltonian graphs that satisfy the condition in Theorem 4 but do not satisfy the condition in Part 1 of Theorem 3 . Similar to the proof of Theorem 2, we need another family of subgraphs of $B_{k}(n)$ defined as follows.

$$
\mathcal{B}_{2}(n, k)=\left\{G \subset B_{k}(n): G=B_{k}(n)-E^{\prime}, \text { where } E^{\prime} \subset E_{1}\left(B_{k}(n)\right) \text { with }\left|E^{\prime}\right|=\left\lfloor\frac{k^{2}}{4}\right\rfloor+1\right\} .
$$

Ideas from 8 together with the following lemma prove Theorem 4 
Lemma 3 Assume $k>1$ and $n \geq k^{4}+3 k^{3}+5 k^{2}+5 k+4$.

1. For each $G \in \mathcal{B}_{1}(n, k)$, we have $q(G) \geq 2 n-k$.

2. For each $G \in \mathcal{B}_{2}(n, k)$, we have $q(G)<2 n-k$.

The paper is organized as follows. In section 2, we will introduce some notation and present necessary preliminary results. We will prove Theorem 2 in section 3 and sketch the proof of Theorem 4 in section 4 . In section 5 , we will construct graphs to show theorems proved in this paper are new and give a few concluding remarks.

\section{Notation and preliminaries}

All graphs in this paper are simple and finite. For those notation not defined here, we refer readers to the monograph written by West [12. For a graph $G$ and $v \in V(G)$, let $d_{G}(v)$ be the degree of $v$ and $N_{G}(v)$ be the neighborhood of $v$, i.e., $N_{G}(v)=\{u \in V(G):\{u, v\} \in E(G)\}$ and $d_{G}(v)=\left|N_{G}(v)\right|$. If the graph $G$ is clear under the context, we will drop the subscript $G$. For a subset $X \subseteq V(G)$, let $G[X]$ be the subgraph of $G$ induced by $X$. We use $A(G)$ to denote the adjacency matrix of $G$. The signless Laplacian matrix $Q(G)$ associated with $G$ is defined as $D+A$, here $D$ is the diagonal matrix of degrees. If $\mathbf{x}$ is a column vector of size $|V(G)|$, then

$$
\langle Q(G) \mathbf{x}, \mathbf{x}\rangle=\sum_{v \in V(G)} d(v) \mathbf{x}_{v}^{2}+2 \sum_{\{u, v\} \in E(G)} \mathbf{x}_{u} \mathbf{x}_{v} .
$$

If $q(G)$ is the largest eigenvalue of $Q(G)$, then by Rayleigh's principle we have

$$
q(G)=\max _{\mathbf{x}} \frac{\langle Q(G) \mathbf{x}, \mathbf{x}\rangle}{\langle\mathbf{x}, \mathbf{x}\rangle} .
$$

Let $\mathbf{f}$ be the eigenvector corresponding to $q(G)$, i.e.,

$$
Q(G) \mathbf{f}=q(G) \mathbf{f} .
$$

By the famous Perron-Frobenius theorem [6], we get $\mathbf{f}_{v}>0$ for each $v \in V(G)$ provided $G$ is connected. By taking the $v$-entry of both sides and rearranging terms, we get

$$
(q(G)-d(v)) \mathbf{f}_{v}=\sum_{u \sim v} \mathbf{f}_{u} .
$$

It is easy to show the following proposition.

Proposition 1 For any $u, v \in V(G)$, we have

$$
(q(G)-d(u))\left(\mathbf{f}_{u}-\mathbf{f}_{v}\right)=(d(u)-d(v)) \mathbf{f}_{v}+\sum_{s \in N(u) \backslash N(v)} \mathbf{f}_{s}-\sum_{t \in N(v) \backslash N(u)} \mathbf{f}_{t} .
$$

Proof: By recalling (1), we get

$$
\begin{aligned}
(q(G)-d(u)) \mathbf{f}_{u} & =\sum_{a \in N(u)} \mathbf{f}_{a}, \\
(q(G)-d(v)) \mathbf{f}_{v} & =\sum_{b \in N(v)} \mathbf{f}_{b} .
\end{aligned}
$$


Therefore,

$$
\begin{aligned}
(q(G)-d(u))\left(\mathbf{f}_{u}-\mathbf{f}_{v}\right) & =(q(G)-d(u)) \mathbf{f}_{u}-(q(G)-d(v)) \mathbf{f}_{v}+(d(u)-d(v)) \mathbf{f}_{v} \\
& =(d(u)-d(v)) \mathbf{f}_{v}+\sum_{s \in N(u)} \mathbf{f}_{s}-\sum_{t \in N(v)} \mathbf{f}_{t} \\
& =(d(u)-d(v)) \mathbf{f}_{v}+\sum_{s \in N(u) \backslash N(v)} \mathbf{f}_{s}-\sum_{t \in N(v) \backslash N(u)} \mathbf{f}_{t} .
\end{aligned}
$$

The proposition is proved.

We will repeatedly use the proposition above. The following theorem from 4 provides an upper bound for $q(G)$.

Theorem 5 Let $G$ be a graph of order $n$. Then

$$
q(G) \leq \frac{2 e(G)}{n-1}+n-2 .
$$

For balanced bipartite graphs, we will apply the following theorem from 8 .

Theorem 6 Let $G$ be a balanced bipartite graph of order $2 n$. Then

$$
q(G) \leq \frac{e(G)}{n}+n .
$$

In the course of proving our results, we will need the following two theorems from $[8]$.

Theorem 7 Let $G$ be a graph of order $n \geq 6 k+5$, where $k \geq 1$. If $\delta(G) \geq k$ and

$$
e(G)>\left(\begin{array}{c}
n-k-1 \\
2
\end{array}\right)+(k+1)^{2},
$$

then $G$ is Hamiltonian unless $G \subseteq L_{k}(n)$ or $G \subseteq M_{k}(n)$.

Theorem 8 Let $G$ be a balanced bipartite graph of order $2 n$. If $\delta(G) \geq k \geq 1, n \geq 2 k+1$ and

$$
e(G)>n(n-k-1)+(k+1)^{2},
$$

then $G$ is Hamiltonian unless $G \subseteq B_{k}(n)$.

\section{Proof of Theorem 2}

We start with this section by proving Lemma 1 .

Proof of Lemma 1; Let $G \in \mathcal{M}_{1}(n, k) \cup \mathcal{L}_{1}(n, k)$. Recall three subsets $X, Y$, and $Z$ of $V(G)$ for both cases. For each case, we define a column vector $\mathbf{h}$ such that $\mathbf{h}_{u}=1$ for all $u \in Y \cup Z$ and $\mathbf{h}_{v}=0$ for all $v \in X$. We note $q\left(\bar{K}_{k}+K_{n-k}\right)=2(n-k-1)$ and $\mathbf{h}$ is the corresponding eigenvector. If $G \in \mathcal{M}_{1}(n, k)$, then we get

$$
\langle Q(G) \mathbf{h}, \mathbf{h}\rangle-\left\langle Q\left(\bar{K}_{k}+K_{n-k}\right) \mathbf{h}, \mathbf{h}\right\rangle=k^{2}-4\left|E^{\prime}\right| \geq 0 .
$$

Similarly, for $G \in \mathcal{L}_{1}(n, k)$, we have

$$
\langle Q(G) \mathbf{h}, \mathbf{h}\rangle-\left\langle Q\left(\bar{K}_{k}+K_{n-k}\right) \mathbf{h}, \mathbf{h}\right\rangle=k-4\left|E^{\prime}\right| \geq 0 .
$$

By Rayleigh's principle, we obtain $q(G) \geq 2(n-k-1)$ in each case.

In order to prove Lemma 2, we need a lower bound on $q(G)$. 
Proposition 2 For each $G \in \mathcal{M}_{2}(n, k) \cup \mathcal{L}_{2}(n, k)$, we have $q(G)>2 n-2 k-3$.

Proof: Suppose $G \in \mathcal{M}_{2}(n, k) \cup \mathcal{L}_{2}(n, k)$. Let $\mathbf{h}$ be the vector defined in the proof of Lemma 1. In the case of $G \in \mathcal{M}_{2}(n, k)$, we have

$$
\langle Q(G) \mathbf{h}, \mathbf{h}\rangle-\left\langle Q\left(\bar{K}_{k}+K_{n-k}\right) \mathbf{h}, \mathbf{h}\right\rangle=k^{2}-4\left|E^{\prime}\right| \geq-4 .
$$

In the case of $G \in \mathcal{L}_{2}(n, k)$, we have

$$
\langle Q(G) \mathbf{h}, \mathbf{h}\rangle-\left\langle Q\left(\bar{K}_{k}+K_{n-k}\right) \mathbf{h}, \mathbf{h}\right\rangle=k-4\left|E^{\prime}\right| \geq-4 .
$$

In each case, we have $q(G) \geq 2(n-k-1)-\frac{4}{\|\mathbf{h}\|^{2}}>2 n-2 k-3$.

We next prove Lemma 2, In the following propositions, we will assume $G \in \mathcal{M}_{2}(n, k)$ and give their detailed proofs. Since arguments in the case of $G \in \mathcal{L}_{2}(n, k)$ are similar to those in the case of $G \in \mathcal{M}_{2}(n, k)$, we will only sketch them.

Let $G$ be a graph from $\mathcal{M}_{2}(n, k)$ with the maximum signless Laplacian spectral radius. Moreover, we assume $G[Y]$ contains the largest number of edges. Let $\mathbf{f}$ be the eigenvector corresponding to $q(G)$. We assume further $\max _{v \in V(G)} \mathbf{f}_{v}=1$. Our goal is to show the vector $\mathbf{f}$ is close (entrywise) to the vector $\mathbf{h}$ as defined in the proof of Lemma 1

We define two subsets of $Y$ as follows:

$$
Y_{1}=\{y \in Y: d(y)=n-1\} \text { and } Y_{2}=\{y \in Y: d(y) \leq n-2\} .
$$

Similarly, we define two subsets of $Z$ as follows:

$$
Z_{1}=\{z \in Z: d(z)=n-k-1\} \text { and } Z_{2}=\{z \in Z: d(z) \leq n-k-2\} .
$$

We note $Z_{1} \neq \emptyset$ as $n \geq k^{4}+k^{3}+4 k^{2}+k+6$. To compare the difference between $\max _{v \in V(G)} \mathbf{f}_{v}$ and $\min _{v \in Y \cup Z} \mathbf{f}_{v}$, we need to prove the following propositions.

Proposition 3 Assume $G \in \mathcal{M}_{2}(n, k)$ as defined above. For each $x \in X$, we have $\mathbf{f}_{x} \leq$ $\frac{k}{q(G)-k}$.

Proof: Applying equation (11) with $x$, we get

$$
(q(G)-d(x)) \mathbf{f}_{x}=\sum_{y \in Y} \mathbf{f}_{y} .
$$

Since $d(x)=k$ and $\max _{v \in V(G)} \mathbf{f}_{v}=1$, the lemma follows.

Proposition 4 Assume $G \in \mathcal{M}_{2}(n, k)$ as defined above. If $Y_{2} \neq \emptyset$, then we have $\mathbf{f}_{y}<\mathbf{f}_{z}$ for all $y \in Y_{2}$ and $z \in Z_{1}$.

Proof: We assume that there are some $y \in Y_{2}$ and $z \in Z_{1}$ such that $\mathbf{f}_{y} \geq \mathbf{f}_{z}$. Let $w$ be a vertex from $Y$ such that $\{y, w\}$ is a non-edge. We define a new graph $G^{\prime} \in \mathcal{M}_{2}(n, k)$ by removing $\{w, z\}$ and adding $\{y, w\}$, as shown in Figure 1. Since

$$
\left\langle Q\left(G^{\prime}\right) \mathbf{f}, \mathbf{f}\right\rangle-\langle Q(G) \mathbf{f}, \mathbf{f}\rangle=\left(\mathbf{f}_{y}-\mathbf{f}_{z}\right)\left(\mathbf{f}_{y}+\mathbf{f}_{z}+2 \mathbf{f}_{w}\right) \geq 0,
$$

we get $q\left(G^{\prime}\right) \geq q(G)$ and $G^{\prime}[Y]$ has more edges than $G[Y]$, which is a contradiction to the choice of $G$. The proposition is proved.

Proposition 5 Assume $G \in \mathcal{M}_{2}(n, k)$ as defined above. If $Z_{2} \neq \emptyset$, then we have $\mathbf{f}_{w}<\mathbf{f}_{z}$ for any $w \in Z_{2}$ and $z \in Z_{1}$. 


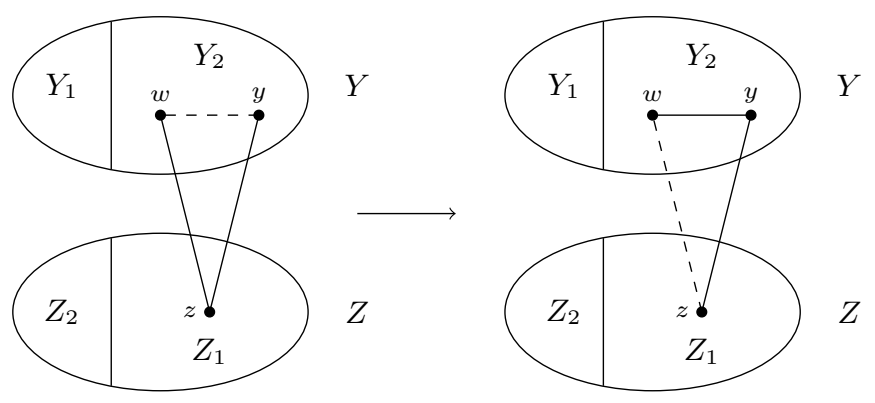

Figure 1: An example for $G$ and $G^{\prime}$

Proof: We first notice $N(w) \backslash\{z\} \subset N(z) \backslash\{w\}$ for each $w \in Z_{2}$ and $z \in Z_{1}$. Applying Proposition 1 with $u=z$ and $v=w$, we get

$$
(q(G)-d(z))\left(\mathbf{f}_{z}-\mathbf{f}_{w}\right)=(d(z)-d(w)) \mathbf{f}_{w}+\mathbf{f}_{w}-\mathbf{f}_{z}+\sum_{s \in N(z) \backslash(N(w) \cup w)} \mathbf{f}_{s} .
$$

Equivalently,

$$
(q(G)-d(z)+1)\left(\mathbf{f}_{z}-\mathbf{f}_{w}\right)=(d(z)-d(w)) \mathbf{f}_{w}+\sum_{s \in N(z) \backslash(N(w) \cup w)} \mathbf{f}_{s} .
$$

We note $d(w)<d(z)$ and the lemma follows.

Similarly, we can use Proposition 1 to show the following one.

Proposition 6 Assume $G \in \mathcal{M}_{2}(n, k)$ as defined above.

1. If $Y_{1}, Y_{2} \neq \emptyset$, then we have $\mathbf{f}_{s}<\mathbf{f}_{t}$ for any $s \in Y_{2}$ and $t \in Y_{1}$.

2. If $Y_{1} \neq \emptyset$, then we have $\mathbf{f}_{z}<\mathbf{f}_{y}$ for any $y \in Y_{1}$ and $z \in Z_{1}$.

The key step for proving Lemma 2 is to show the following proposition.

Proposition 7 Assume $G \in \mathcal{M}_{2}(n, k)$ as defined above. We have

$$
\max _{v \in V(G)} \mathbf{f}_{v}-\min _{u \in Y \cup Z} \mathbf{f}_{u} \leq \frac{k^{2}+2 k+6}{2(q(G)-n+1)} .
$$

Proof: We have two cases depending on $Y_{1}$.

Case 1: $Y_{1}=\emptyset$. By Proposition 4 and Proposition [5] we get $\max _{v \in V(G)} \mathbf{f}_{v}$ is attained by vertices from $Z_{1}$. Take $z$ as an arbitrary vertex from $Z_{1}$. We observe that $z$ is adjacent to all other vertices in $Y \cup Z$.

If $w \in Z_{2}$, then we have $N(z) \backslash(N(w) \cup w)=\{k: k \in Y \cup Z$ and $k \not w\}$. Thus we get $d(z)-d(w) \leq\left\lfloor\frac{k^{2}}{4}\right\rfloor+1$ and $|N(z) \backslash(N(w) \cup w)| \leq\left\lfloor\frac{k^{2}}{4}\right\rfloor+1$. Recalling (2), we get

$$
\begin{aligned}
(q(G)-d(z)+1)\left(\mathbf{f}_{z}-\mathbf{f}_{w}\right) & =(d(z)-d(w)) \mathbf{f}_{w}+\sum_{s \in N(z) \backslash(N(w) \cup w)} \mathbf{f}_{s} \\
& \leq \frac{k^{2}}{2}+2 .
\end{aligned}
$$


Since $d(z)=n-k-1$, we get

$$
\mathbf{f}_{z}-\mathbf{f}_{w} \leq \frac{k^{2}+4}{2(q(G)-n+k+2)}<\frac{k^{2}+2 k+6}{2(q(G)-n+1)}
$$

If $w \in Y_{2}$, then we have $N(z) \backslash(N(w) \cup w)=\{k: k \in Y \cup Z$ and $k \nsim w\}$ and $N(w) \backslash(N(z) \cup z)=X$. Thus $|N(z) \backslash(N(w) \cup w)| \leq\left\lfloor\frac{k^{2}}{4}\right\rfloor+1$. We also observe $|d(z)-d(w)| \leq\left\lfloor\frac{k^{2}}{4}\right\rfloor+k+1$. Applying Proposition 1 with $u=z$ and $v=w$, we get

$$
\begin{aligned}
(q(G)-d(z))\left(\mathbf{f}_{z}-\mathbf{f}_{w}\right) & =(d(z)-d(w)) \mathbf{f}_{w}+\mathbf{f}_{w}+\sum_{s \in N(z) \backslash(N(w) \cup w)} \mathbf{f}_{s}-\mathbf{f}_{z}-\sum_{t \in X} \mathbf{f}_{t} \\
& \leq\left\lfloor\frac{k^{2}}{4}\right\rfloor+k+1+\mathbf{f}_{w}+\left\lfloor\frac{k^{2}}{4}\right\rfloor+1-\mathbf{f}_{z}-\sum_{t \in X} \mathbf{f}_{t} .
\end{aligned}
$$

Equivalently,

$$
(q(G)-d(z)+1)\left(\mathbf{f}_{z}-\mathbf{f}_{w}\right) \leq \frac{k^{2}}{2}+k+2
$$

We get

$$
\mathbf{f}_{z}-\mathbf{f}_{w} \leq \frac{k^{2}+2 k+4}{2(q(G)-n+k+2)}<\frac{k^{2}+2 k+6}{2(q(G)-n+1)}
$$

Case 2: $Y_{1} \neq \emptyset$. By Proposition $\left[5\right.$ and Proposition [6] we get $\max _{v \in V(G)} \mathbf{f}_{v}$ is attained by vertices from $Y_{1}$. Let $z$ be a vertex from $Y_{1}$. By repeating the argument in Case 1, we can show

$$
\mathbf{f}_{z}-\mathbf{f}_{w} \leq \frac{k^{2}+2 k+6}{2(q(G)-n+2)}<\frac{k^{2}+2 k+6}{2(q(G)-n+1)}
$$

for any $w \in Y_{2} \cup Z$.

The proof is completed.

We next assume $G \in \mathcal{L}_{2}(n, k)$. Recalling three subsets $X, Y$, and $Z$ of $V(G)$, here we suppose $Y=\{y\}$. We take such $G$ satisfying $q(G)$ is maximum and $d(y)$ is the largest. Let $\mathbf{f}$ be the eigenvector corresponding to $q(G)$. Suppose $\mathbf{f}$ satisfies $\max _{v \in V(G)} \mathbf{f}_{v}=1$. We define

$$
Z_{1}=\{z \in Z: d(z)=n-k-1\} \text { and } Z_{2}=\{z \in Z: d(z) \leq n-k-2\} .
$$

We will need the following proposition for the case of $G \in \mathcal{L}_{2}(n, k)$.

Proposition 8 Let $G$ be assumed as above.

1. For each $x \in X$, we have $\mathbf{f}_{x} \leq \frac{k}{q(G)-k}$.

2. If $Z_{2} \neq \emptyset$, then we have $\mathbf{f}_{w}<\mathbf{f}_{z}$ for each $w \in Z_{2}$ and each $z \in Z_{1}$.

3. If $d(y) \leq n-2$, then $\mathbf{f}_{y}<\mathbf{f}_{z}$ for each $z \in Z_{1}$.

4. If $d(y)=n-1$, then $\mathbf{f}_{y}>\mathbf{f}_{z}$ for each $z \in Z_{1}$.

5. We have $\max _{v \in V(G)} \mathbf{f}_{v}-\min _{u \in Y \cup Z} \mathbf{f}_{u} \leq \frac{k^{2}+2 k+6}{2(q(G)-n+1)}$.

Proof: Since proofs of parts of this proposition are very similar to those of Propositions 3 , 4 45, 6. 7] we only give the sketch here. Parts 1, 2, 3, 4 use ideas from proofs of Propositions 3, 5, 4, 7 respectively. For Part 5, we have two cases depending on $d(y)$. 
Case 1: $d(y) \leq n-2$. We note $\max _{v \in V(G)} \mathbf{f}_{v}$ is achieved by vertices from $Z_{1}$. Let $z$ be an arbitrary vertex from $Z_{1}$.

If $w \in Z_{2}$, then we have $N(z) \backslash(N(w) \cup w)=\{k: k \in Y \cup Z$ and $k \nsim w\}$. Therefore, $|N(z) \backslash(N(w) \cup w)| \leq\left\lfloor\frac{k}{4}\right\rfloor+1$ and $d(z)-d(w) \leq\left\lfloor\frac{k}{4}\right\rfloor+1$. By Proposition 1 we get

$$
(q(G)-d(z))\left(\mathbf{f}_{z}-\mathbf{f}_{w}\right)=(d(z)-d(w)) \mathbf{f}_{w}+\mathbf{f}_{w}+\sum_{s \in N(z) \backslash(N(w) \cup w)} \mathbf{f}_{s}-\mathbf{f}_{z} .
$$

Therefore,

$$
(q(G)-d(z)+1)\left(\mathbf{f}_{z}-\mathbf{f}_{w}\right) \leq \frac{k}{2}+2 .
$$

If $w=y$, then we have $N(z) \backslash(N(w) \cup w)=\{k: k \in Y \cup Z$ and $k \not w\}$ and $N(w) \backslash(N(z) \cup z)=X$. We notice $|d(z)-d(w)| \leq\left\lfloor\frac{k}{4}\right\rfloor+k+1$ and $|N(z) \backslash(N(w) \cup w)| \leq$ $\left\lfloor\frac{k}{4}\right\rfloor+1$. By Proposition 1 , we get

$$
\begin{aligned}
(q(G)-d(z))\left(\mathbf{f}_{z}-\mathbf{f}_{w}\right) & =(d(z)-d(w)) \mathbf{f}_{w}+\mathbf{f}_{w}+\sum_{s \in N(z) \backslash(N(w) \cup w)} \mathbf{f}_{s}-\mathbf{f}_{z}-\sum_{t \in X} \mathbf{f}_{t} \\
& \leq\left\lfloor\frac{k}{4}\right\rfloor+k+1+\mathbf{f}_{w}+\left\lfloor\frac{k}{4}\right\rfloor+1-\mathbf{f}_{z}-\sum_{t \in X} \mathbf{f}_{t} .
\end{aligned}
$$

Therefore,

$$
(q(G)-d(z)+1)\left(\mathbf{f}_{z}-\mathbf{f}_{w}\right) \leq \frac{k}{2}+k+1 .
$$

In both subcases, we have $\mathbf{f}_{z}-\mathbf{f}_{w} \leq \frac{k^{2}+2 k+6}{2(q(G)-n+1)}$.

Case 2: $d(y)=n-1$. We note $\max _{v \in V(G)} \mathbf{f}_{v}=\mathbf{f}_{y}$. We can use the argument above to show the desired upper bound on $\mathbf{f}_{y}-\mathbf{f}_{z}$ for all $z \in Z$.

The proposition is proved.

We are ready to prove Lemma 2 ,

Proof of Lemma 2, We first assume $G \in \mathcal{M}_{2}(n, k)$ such that $G$ has the largest signless Laplaican spectral radius and $G[Y]$ contains the largest number of edges. Let $q(G)$ be the largest eigenvalue of $Q(G)$ and $\mathbf{f}$ be the eigenvector of $q(G)$. Recalling Proposition 3 and Proposition 7 we get

$$
\begin{aligned}
\langle Q(G) \mathbf{f}, \mathbf{f}\rangle-\left\langle Q\left(\bar{K}_{k}+K_{n-k}\right) \mathbf{f}, \mathbf{f}\right\rangle & =\sum_{x \in X, y \in Y}\left(\mathbf{f}_{x}+\mathbf{f}_{y}\right)^{2}-\sum_{\{u, v\} \in E^{\prime}}\left(\mathbf{f}_{u}+\mathbf{f}_{v}\right)^{2} \\
& \leq k^{2}\left(1+\frac{k}{q(G)-k}\right)^{2}-4\left|E^{\prime}\right|\left(1-\frac{k^{2}+2 k+6}{2(q(G)-n+1)}\right)^{2} \\
& <0 .
\end{aligned}
$$

Here we used $n \geq k^{4}+k^{3}+4 k^{2}+k+6$ and $q(G)>2 n-2 k-3$ by Proposition 2. We note $q\left(\bar{K}_{k}+K_{n-k}\right)=2(n-k-1) \geq\left\langle Q\left(\bar{K}_{k}+K_{n-k}\right) \mathbf{f}, \mathbf{f}\right\rangle /\langle\mathbf{f}, \mathbf{f}\rangle$. Thus we proved $q(G)<2(n-k-1)$ in this case.

When $G \in \mathcal{L}_{2}(n, k)$, we can assume $q(G)$ is the maximum and $d(y)$ is the largest. Applying Proposition 8 and repeating the argument for $G \in \mathcal{M}_{2}(n, k)$, we can prove $q(G)<2(n-k-1)$ easily.

We are ready to prove Theorem 2

Proof of Theorem 2; By theorem [5, we get

$$
2(n-k-1) \leq q(G) \leq \frac{2 e(G)}{n-1}+n-2 .
$$


Since we assume $n \geq k^{4}+k^{3}+4 k^{2}+k+6$, we get

$$
\begin{aligned}
e(G) & >\frac{(n-2 k)(n-1)}{2} \\
& =\left(\begin{array}{c}
n-k-1 \\
2
\end{array}\right)+\frac{2 n-k^{2}-k-2}{2} \\
& \geq\left(\begin{array}{c}
n-k-1 \\
2
\end{array}\right)+(k+1)^{2} .
\end{aligned}
$$

By Theorem 7, $G$ has a Hamilton cycle unless $G \subseteq L_{k}(n)$ or $G \subseteq M_{k}(n)$. Together with Lemma 1 and Lemma 2, we complete the proof.

\section{Proof of Theorem 4}

We first observe the following: if Lemma 3 holds, then the combination of Theorem 6 and Theorem 8 yields Theorem 4 . We are left to prove Lemma 3.

We note $q\left(K_{n, n-k}+\overline{K_{k}}\right)=2 n-k$. Let $\mathbf{f}$ be an eigenvector corresponding to $2 n-k$. If we assume $\max _{v} \mathbf{f}_{v}=1$, then we have $\mathbf{f}_{u}=1$ for $d(u)=n$ and $\mathbf{f}_{u}=1-\frac{k}{n}$ for $d(u)=n-k$. Recalling the definition of $\mathcal{B}_{1}(n, k)$ and repeating the proof for Lemma 1, we can prove Part 1 of Lemma 3. Given Proposition 9, we can show Part 2 of Lemma 3 by the same argument as the one in the proof of Lemma 2 ,

Let $G \in \mathcal{B}_{2}(n, k)$ such that $q(G)$ is the maximum and $G[Y \cup Z]$ induces the largest number of edges. We assume the corresponding eigenvector is $\mathbf{f}$ and $\max _{v} \mathbf{f}_{v}=1$. We define $Y_{1}=\{v \in Y: d(v)=n\}, Y_{2}=\{v \in Y: d(v) \leq n-1\}, W_{1}=\{v \in W: d(v)=n-k\}$, $W_{2}=\{v \in W: d(v) \leq n-k-1\}, Z_{1}=\{v \in Z: d(v)=n\}$, and $Z_{2}=\{v \in Z: d(v) \leq n-1\}$.

It remains to establish the following proposition.

Proposition 9 Let $G$ be assumed as above.

1. $2 n-k-1 \leq q(G) \leq 2 n-k+1$.

2. For each $x \in X$, we have $\mathbf{f}_{x} \leq \frac{k}{q(G)-k}$.

3. If $Y_{2} \neq \emptyset$, then we have $\mathbf{f}_{y}<\mathbf{f}_{w}$ for each $y \in Y_{2}$ and each $w \in W_{1}$.

4. If $Y_{1} \neq \emptyset$, then we have $\mathbf{f}_{w}<\mathbf{f}_{y}$ for each $y \in Y_{1}$ and each $w \in W$.

5. If $Y_{1}, Y_{2} \neq \emptyset$, then we have $\mathbf{f}_{t}<\mathbf{f}_{y}$ for each $t \in Y_{2}$ and each $y \in Y_{1}$.

6. If $W_{2} \neq \emptyset$, then we have $\mathbf{f}_{s}<\mathbf{f}_{t}$ for each $s \in W_{2}$ and each $t \in W_{1}$.

7. If $Z_{2} \neq \emptyset$, then we have $\mathbf{f}_{t}<\mathbf{f}_{z}$ for each $t \in Z_{2}$ and each $z \in Z_{1}$.

8. We have $\max _{v \in V(G)} \mathbf{f}_{v}-\min _{u \in Y \cup Z \cup W} \mathbf{f}_{u} \leq \frac{3 k^{2}+8 k+20}{4(q(G)-n)}$.

Proof: The upper bound in Part 1 follows from Theorem 6 and the proof of the lower bound uses the same idea as the one in Proposition 2, Equation (11) gives Part 2. When we prove Part 3, we have to apply Proposition 1 and the edge-switching idea as we did in the proof of Proposition 4. Part 4-Part 7 follow from Proposition 1 straightforwardly.

For the proof of part 8, we consider the following cases. 
Case 1: $Y_{1}=\emptyset$. We observe $\max _{v \in V(G)} \mathbf{f}_{v}$ is achieved by vertices from $W_{1} \cup Z_{1}$. Suppose $\mathbf{f}_{w}=\max _{v \in V(G)} \mathbf{f}_{v}$ for some $w \in W_{1}$. We note $\left|W_{1}\right| \geq n-\left\lfloor\frac{k^{2}}{4}\right\rfloor-1$ and $\mathbf{f}_{w}=\mathbf{f}_{w^{\prime}}$ for all $w, w^{\prime} \in W_{1}$. Let $z \in Z$. We have $d(z) \leq n$. By equation (11), we get $(q(G)-d(z)) \mathbf{f}_{z}=$ $\sum_{v: v \in N(z)} \mathbf{f}_{v}$. Obviously, we have $(q(G)-d(z)) \mathbf{f}_{z} \geq \sum_{w \in W_{1}} \mathbf{f}_{w}$. Therefore,

$$
\mathbf{f}_{z} \geq \frac{4 n-k^{2}-4}{4(q(G)-n)} .
$$

Let $y$ be an arbitrary vertex from $(Y \cup W) \backslash W_{1}$. We can apply Proposition 1 with $u=w$ and $v=y$ to show

$$
\mathbf{f}_{z}-\mathbf{f}_{y} \leq \frac{k^{2}+4 k+4}{2(q(G)-n+1)}
$$

If $\mathbf{f}_{z}=\max _{v \in V(G)} \mathbf{f}_{v}$ for some $z \in Z_{1}$, then we can use equation (1) to show a lower bound on $\mathbf{f}_{y}$ for each $y \in Y \cup W$. We apply Proposition 1 to get a lower bound on $\mathbf{f}_{z^{\prime}}$ for all $z^{\prime} \in Z_{2}$. Tedious calculation can confirm Part 8 in this case.

Case 2: $Y_{1} \neq \emptyset$. We observe $\max _{v \in V(G)} \mathbf{f}_{v}$ is achieved by vertices from $Y_{1} \cup Z_{1}$. We can repeat the argument in Case 1 to show Part 8 in this case.

We completed the proof of this proposition.

\section{Concluding remarks and examples}

In this paper, we proved a new sufficient spectral condition for the Hamiltonicity of graphs. The main work is to prove a lower bond on the difference between the largest entry and the smallest entry (of a particular subset of vertices) of the principal eigenvector of a family of graphs. We mention here that the idea in the proof of Lemma 2 can be used to give an alternative proof for Theorem 6 in [10].

We next construct a graph that is a variant of $M_{k}(n)$. For the graph $M_{k}(n)$, we recall three subsets of vertices $X, Y$, and $Z$. For $k \geq 3$ and $n \geq 2 k+1$, let $M_{k}^{\prime}(n)$ be a graph obtained from $M_{k}(n)$ by connecting two vertices from $X$ and deleting two edges with endpoints from $Z$. We observe $M_{k}^{\prime}(n)$ is Hamiltonian. One can easily show $\lambda\left(M_{k}^{\prime}(n)\right)<n-k-1$ and $q\left(M_{k}^{\prime}(n)\right) \geq 2(n-k-1)$ for $n$ large enough. Therefore, we are not able to use Theorem 1 to determine whether it contains a Hamiltonian cycle. However, it satisfies the condition in Theorem 4 and we can tell it is Hamiltonian. Similarly, we construct a graph that is a variant of $B_{k}(n)$. For $k \geq 3$ and $n \geq 2 k$, recall subsets $X, Y, Z$, and $W$ of the vertex set of $B_{k}(n)$. Starting from $B_{k}(n)$, we connect two vertices in $X$ and delete two edges from $E(Y \cup W, Z)$. Let $B_{k}^{\prime}(n)$ be the resulting graph. It is not hard to check $\lambda\left(B_{k}^{\prime}(n)\right)<\lambda\left(B_{k}(n)\right)$. Thus we are not able to use Theorem 3 to determine the Hamiltonicity of $B_{k}^{\prime}(n)$. Since one can check $q\left(B_{k}^{\prime}(n)\right) \geq 2 n-k$ easily, Theorem 4 implies that it is Hamiltonian. In conclusion, Theorem 2 and Theorem 4 provide new power for one to determine the Hamiltonicity of graphs with large minimum degree.

\section{References}

[1] V. Benediktovich, Sufficient spectral condition for Hamiltonicity of a graph, Dokl. Nats. Akad. Nauk Belarusi, 59 (2015), 5-12.

[2] S. Butler and F. Chung, Small spectral gap in the combinatorial Laplacian implies Hamiltonian, Ann. Comb., 13(4) (2010), 403-412. 
[3] G. Dirac, Some theorems on abstract graphs, Proc. London Math. Soc. Ser. 3, (2nd ed.), 2(1952), 69-81.

[4] L. Feng and G. Yu, On three conjectures involving the signless Laplacian spectral radius of graphs, Publ. Inst. Math., 85(99) (2009), 35-38.

[5] M. Fiedler and V. Nikiforov, Spectral radius and Hamiltonicity of graphs, Linear Algebra Appl., 432(9) (2010), 2170-2173.

[6] C. Godsil, G. F. Royle, Algebraic Graph Theory, Springer, New York, 2001.

[7] R. Karp, Reducibility among combinatorial problems, in: Complexity of Computer Computations, Plenum Press, New York, 1972, 85-103.

[8] B. Li and B. Ning, Spectral analogues of Erdős's and Moon-Moser's theorems on Hamilton cycles, Linear Multilinear Algebra, 64(11) (2016), 1-18.

[9] M. Lu, H. Liu, and F. Tian, Spectral radius and Hamiltonian graphs, Linear Algebra Appl., 437(7) (2012), 2670-2174.

[10] V. Nikiforov, Spectral radius and Hamiltonicity of graphs with large minimum degree, Czechoslovak Math. J., 66(141) (2016), 925C940.

[11] B. Ning and J. Ge, Spectral radius and Hamiltonian properties of graphs, Linear Multilinear Algebra, 63(8) (2015), 1520C1530.

[12] D. West, Introduction to graph theory. Prentice Hall, Inc., Upper Saddle River, NJ, 1996.

[13] B. Zhou, Signless Laplacian spectral radius and Hamiltonicity, Linear Algebra Appl., 432(2-3) (2010), 566-570. 